\title{
COMPARACIÓN DEL ESTADIO FETAL OBTENIDO POSTMORTEM MEDIANTE DOS METODOS ANTROPOMETRICOS
}

\author{
Mirta M. Aliendo*, Emanuel Repetto \\ Cátedra e Instituto de Anatomía Normal, Facultad de Ciencias Médicas, Universidad \\ Nacional de Córdoba, Córdoba, Argentina
}

\begin{abstract}
RESUMEN
En la etapa fetal se observa un rápido incremento de la masa corporal y de todas las dimensiones. La literatura evidencia discrepancias sobre los criterios para determinar el estadio fetal post-mortem, en relación a los parámetros morfométricos utilizados, por lo que nuestro objetivo fue comparar la medición morfológica directa (vertex-coxis, tabla de Hansmann) con la ultrasonografía (medición del fémur), para establecer el grado de confiabilidad en la determinación post-mortem del estadío fetal. Se utilizaron 120 fetos: 1 ) grupo A (60 fetos) estadificado ecográficamente y 2) grupo B (60 fetos) estadificado por tabla de Hansmann. A ambos grupos se le realizaron múltiples mediciones siguiendo parámetros probados según la literatura internacional. Se utilizó calibre de precisión. Parámetros evaluados: longitud vertex-coxis, circunferencia cefálica, diámetro cefálico occipito-frontal, biparietal, longitud mentón-vertex, perímetro toráxico-transverso, circunferencia abdominal y longitudes de brazo, antebrazo, mano, muslo, pierna y pie. Estos valores fueron agrupados por semanas, obteniéndose la media y aplicándose la prueba $t$ de Student. Los resultados demostraron que la diferencia entre los parámetros medidos en el grupo A y en el grupo B eran significativas en todas las semanas, por lo que se observa disparidad en la determinación del estadio fetal por ecografía y los registros correspondientes a la medición vertex-coxis (tabla de Hansmann) postmortem. Concluímos que los resultados obtenidos por ambas modalidades de medición son diferentes para una misma edad gestacional y, por ende, resultaría más apropiado referirse a fetos con ciertas dimensiones según alguno de estos parámetros que a "edad gestacional".
\end{abstract}

Palabras claves: antropometría, biometría fetal, edad gestacional.

\begin{abstract}
In fetal stage, body mass and measurements quickly increase. Scientific literature shows differences on the criteria to determine the post-mortem fetal stage, depending on morphometric parameters. Our objective was to compare both methods, direct morphologic measures (crown-rump length, Hansmann table) and ultrasonography (femur measurement), to establish their reliability on post-mortem determination of fetal age. One hundred and twenty fetuses were studied: 1) group A (60 fetuses) sonographically staged and 2) group B (60 fetuses) staged according to Hansmann table. Many measurements were performed on both groups, following internationally determined parameters. We used a precision gauge. Considered parameters were: crown-rump length, head circumference, occipito-frontal diameter, bi-parietal length, chin-vertex length, thoracic transverse perimeter, abdominal circumference, arm, forearm, hand, thigh, leg and foot lengths. Obtained information was grouped by weeks. We calculated the data mean and significant difference was managed by Student $\mathrm{t}$ test. Results demonstrated significant difference in the considered parameters between group $A$ and $B$, and then, to determine the fetal age.We conclude that results obtained by both measuring modalities were different for the same gestational age, and therefore, it should be more appropriate to consider fetuses by measures obtained following certain parameters than by "gestational age".
\end{abstract}

Key words: anthropometry, fetal biometry, gestational age

\footnotetext{
* Correspondencia a: Dra. Mirta Malvina Aliendo, Clemente Zarraga 1474 Bella Vista, CP 5.000, Córdoba, Argentina. malvialiendo@yahoo.com.ar
}

Recibido: 26 de enero de 2011. Revisado: 09 de febrero de 2011. Aceptado: 19 de febrero de 2011. 


\section{INTRODUCCIÓN}

Existen diversas tablas antropométricas, que aplican diferentes métodos, para estudiar las variables morfométricas relacionadas a la determinación de la edad gestacional postmortem (Hansmann, 1979; Deter, 1982; Leung, 2008).

La utilización de la ecografía para la establecer la edad gestacional intrauterina ha modificado los criterios tradicionales y aporta un nuevo enfoque, aplicable también a la determinación postmortem.

En la etapa fetal (de la novena semana de gestación al nacimiento), la característica principal es el rápido incremento de la masa corporal y de todas sus dimensiones.

La longitud del feto suele expresarse como longitud vertex-coxis o vertex-talón. Estas medidas expresadas en centímetros se correlacionan con la edad del feto expresada en semanas o meses (Sadler, 2003).

Con frecuencia se ha considerado que el periodo fetal es un tiempo de crecimiento y maduración fisiológica de los sistemas orgánicos, y no ha recibido mucha atención por la embriología tradicional. Sin embargo, los avances en radiología y en otras técnicas diagnósticas han mejorado mucho el acceso al feto y en este momento resulta posible determinar el patrón de crecimiento con un grado notable de precisión (Carlson, 2005).

Existen discrepancias para determinar la edad gestacional post-mortem en cuanto a los parámetros morfométricos utilizados, por lo que nuestro objetivo es cotejar la edad gestacional obtenida a través de la medición morfológica directa (vertex-coxis) expresado en las tablas de Hansmann (Lapunzina, 2002) con la edad gestacional obtenida por medio de la ultrasonografía a través de la medición del fémur, comparando las medias de los diversos parámetros morfométricos (mediciones externas cuantificadas) obtenidas en cada grupo.

Planteamos una hipótesis nula para la cual no existen diferencias estadísticamente significativas entre ambos grupos, es decir, utilizando ambos métodos de medición obtenemos los mismos resultados; y proponiendo como hipótesis experimental que existen diferencias significativas entre ambos grupos.

Así, el objetivo es comparar la medición morfológica directa (vertex-coxis, tabla de Hansmann) con la ultrasonografía (medición del fémur), para establecer el grado de confiabilidad en la determinación post-mortem del estadío fetal.

\section{MATERIAL Y METODOS}

El estudio se realizó en fetos obtenidos de abortos espontáneos en el Hospital Universitario de Maternidad, de la Ciudad de Córdoba, Argentina. Se obtuvieron 120 fetos normales y en excelente estado de conservación, bien hidratados, sin laceraciones ni retracciones, no deformados y sin hematomas ni edemas. No se incluyeron en el estudio fetos malformados ni aquellos normales pero en mal estado de conservación, mutilados, deformados o macerados.

Estos 120 especímenes se obtuvieron en dos etapas: primero 60 fetos cuya edad gestacional se encontraba determinada previamente por medición ecográfica del fémur y en una segunda etapa 60 fetos cuya edad gestacional determinamos por medición directa vertex-coxis (Tabla de Hansmann).

Los fetos fueron conservados, por inmersión, en solución acuosa de formol al $4 \%$.

Se determinaron 2 grupos de 60 fetos cada uno: A) con edad gestacional determinada por ecografía y B) con edad gestacional determinada por medición vertex-coxis (tabla de Hansmann). A ambos grupos se le efectuó la medición de todos los parámetros que se citan a continuación, para realizar el estudio comparativo de los resultados.

Para asegurar la precisión y fiabilidad de los datos presentados se decidió la participación en las mediciones de un solo investigador y el empleo de un único dispositivo de medida.

Para la medición de los diferentes parámetros se utilizó un calibre de precisión. Las circunferencias se tomaron con hilo de nylon monofilamento para que se adaptara a la superficie fetal y luego se procedió a la medición del segmento correspondiente.

Las mediciones externas cuantificadas fueron:

- Longitud vertex-coxis

- Diámetros cefálicos:

- circunferencia cefálica a nivel del piso medio craneal

- occipito-frontal

- bi-parietal

- mentón-vertex

- Perímetros fetales:

- toráxico transverso

- circunferencia abdominal (inmediatamente por arriba del cordón umbilical)

- Longitud del miembro superior

- Brazo (del tubérculo mayor del húmero hasta la parte distal del codo)

- Antebrazo (del pliegue del codo hasta el pliegue de flexión de la muñeca) 
- Mano (del pliegue de flexión de la muñeca hasta el extremo del dedo medio)

- Longitud del miembro inferior:

- Muslo (del trocánter mayor del fémur hasta los cóndilos femorales)

- Pierna (del platillo tibial hasta la planta del pie)

- Pie (desde el talón hasta el extremo del dedo más largo)

Se utilizó el software SPSS Statistics Versión para organizar, resumir y expresar en tablas la información recolectada de cada grupo.

Dicho software aplica la prueba t de Student para datos independientes, utilizada para comparar medias de variables en dos grupos de casos que son independientes entre sí.

Es el sistema recomendado por la Organización Mundial de la Salud para comparar mediciones antropométricas individuales en referencia a la población (Salomon, 2006).

Al hacer la comparación de medias se tiene en cuenta otro factor, que es la igualdad o no de las varianzas en los dos grupos, pues los resultados no serán iguales en ambos casos. Si asumimos igualdad de varianzas podremos calcular un estimador único y más estable de la varianza poblacional a partir de las varianzas muestrales. $\mathrm{Si}$ las varianzas no son iguales no podremos hacer esto. Por ello se utiliza el test de hipótesis para la igualdad de las varianzas (test de Levene), ya que si se estiman las varianzas como iguales se usa un procedimiento estadístico para calcular t; y si las varianzas son distintas se utiliza una variante en la fórmula.

El software se utilizó para identificar si las diferencias son 0 no significativas pues nos entrega el valor de la probabilidad $(P)$ para el valor t obtenido.

\section{RESULTADOS}

Los resultados obtenidos permitieron descartar la hipótesis nula y afirmar la hipótesis experimental, en donde se verifica que la diferencia entre los parámetros medidos en el grupo en el cual la edad gestacional fue determinada por medición ecográfica del fémur y el grupo en el cual la edad gestacional fue determinada a través de la tabla de Hansmann (longitud vertex-coxis) era significativa en todas las semanas.

\begin{tabular}{|c|c|c|c|c|c|c|c|c|}
\hline \multirow{3}{*}{$\begin{array}{l}\text { Edad gestacional } \\
\text { (semanas) }\end{array}$} & \multicolumn{8}{|c|}{ Brazo } \\
\hline & \multicolumn{3}{|c|}{ USG } & \multicolumn{3}{|c|}{ Hansmann } & \multirow{2}{*}{$T$} & \multirow{2}{*}{$\mathrm{P}$} \\
\hline & $\mathrm{n}$ & $\mathrm{M}$ & $S$ & $\mathrm{n}$ & $M$ & $S$ & & \\
\hline 14 & 10 & 2,503 & 229 & 13 & 1,440 & 118 & 13,309 & ,000 \\
\hline 15 & 6 & 3,335 & ,069 & 8 & 1,835 & 166 & 20,543 & ,000 \\
\hline 16 & 9 & 3,903 & 117 & 5 & 2,204 & ,065 & 29,508 & ,000 \\
\hline 17 & 9 & 4,174 & 444 & 8 & 2,852 & ,358 & 6,689 & ,000 \\
\hline 18 & 9 & 4,660 & 358 & 9 & 3,357 & 183 & 9,705 & ,000 \\
\hline 19 & 6 & 4,946 & ,084 & 7 & 3,670 & 180 & 5,775 & ,000 \\
\hline 20 & 4 & 5,825 & 140 & 5 & 4,204 & 248 & 11,535 & ,000 \\
\hline 21 & 7 & 6,084 & ,244 & 5 & 4,444 & 135 & 13,473 & ,000 \\
\hline
\end{tabular}

Tabla 1: USG: ultrasonografía, $\mathbf{n}: \mathrm{N}^{\circ}$ de individuos, M: Media, S: Desvío Standard. T: Prueba $t$ de Student, P: Probabilidad. Numeración en rojo: valores a los que se les aplicó el test de Levene. 


\begin{tabular}{|c|c|c|c|c|c|c|c|c|}
\hline \multirow{2}{*}{$\begin{array}{c}\text { Edad gestacional } \\
\text { (semanas) }\end{array}$} & \multicolumn{7}{|c|}{ Muslo } \\
\cline { 2 - 9 } & $\mathrm{n}$ & $\mathrm{M}$ & $\mathrm{S}$ & $\mathrm{n}$ & $\mathrm{M}$ & $\mathrm{S}$ & $\mathrm{T}$ & $\mathrm{P}$ \\
\hline 14 & 10 & 2,501 &, 228 & 13 & 1,330 &, 063 & 15,753 &, 000 \\
\hline 15 & 6 & 3,441 &, 079 & 8 & 1,563 &, 122 & 32,535 &, 000 \\
\hline 16 & 9 & 3,800 &, 166 & 5 & 1,844 &, 048 & 32,876 &, 000 \\
\hline 17 & 9 & 4,327 &, 254 & 8 & 2,313 &, 282 & 15,463 &, 000 \\
\hline 18 & 9 & 4,771 &, 278 & 9 & 2,848 &, 164 & 17,833 &, 000 \\
\hline 19 & 6 & 4,961 &, 132 & 7 & 3,541 &, 330 & 9,825 &, 000 \\
\hline 20 & 4 & 5,137 &, 211 & 5 & 4,020 &, 111 & 10,273 &, 000 \\
\hline 21 & 7 & 6,044 &, 559 & 5 & 4,474 &, 18407 & 5,978 &, 000 \\
\hline
\end{tabular}

Tabla 2: USG: ultrasonografía, $\mathbf{n}$ : $\mathrm{N}^{\circ}$ de individuos, M: Media, S: Desvío Standard. T: Prueba t de Student, P: Probabilidad. Numeración en rojo: valores a los que se les aplicó el test de Levene.

\begin{tabular}{|c|c|c|c|c|c|c|c|c|}
\hline \multirow{2}{*}{$\begin{array}{c}\text { Edad gestacional } \\
(\text { semanas })\end{array}$} & \multicolumn{7}{|c|}{ Vertex - coxis } \\
\cline { 2 - 9 } & \multicolumn{3}{|c|}{ USG } & \multicolumn{3}{|c|}{ Hansmann } & \multirow{2}{*}{$\mathrm{P}$} \\
\cline { 2 - 9 } & $\mathrm{n}$ & $\mathrm{M}$ & $\mathrm{S}$ & $\mathrm{N}$ & $\mathrm{M}$ & $\mathrm{S}$ & $\mathrm{T}$ & \\
\hline 14 & 10 & 10,501 &, 300 & 13 & 9,620 &, 330 & 6,582 &, 000 \\
\hline 15 & 6 & 11,166 &, 111 & 8 & 10,560 &, 326 & 4,894 &, 001 \\
\hline 16 & 9 & 11,993 &, 304 & 5 & 11,290 &, 053 & 6,741 &, 000 \\
\hline 17 & 9 & 13,936 &, 673 & 8 & 11,610 &, 186 & 9,948 &, 000 \\
\hline 18 & 9 & 16,058 &, 249 & 9 & 12,171 &, 361 & 26,547 &, 000 \\
\hline 19 & 6 & 16,775 &, 293 & 7 & 12,900 &, 249 & 25,751 &, 000 \\
\hline 20 & 4 & 18,322 &, 566 & 5 & 13,318 &, 093 & 17,465 &, 000 \\
\hline 21 & 7 & 19,054 &, 166 & 5 & 13,590 &, 168 & 55,892 &, 000 \\
\hline
\end{tabular}

Tabla 3- USG: ultrasonografía, $\mathbf{n}$ : $\mathrm{N}^{\circ}$ de individuos, M: Media, S: Desvío Standard. T: Prueba $t$ de Student, P: Probabilidad. Numeración en rojo: valores a los que se les aplicó el test de Levene 
Analizando los resultados se pudo verificar que el parámetro de la longitud del brazo (tabla 1) y del muslo (tabla 2) dieron en todas las semanas una $P=0,000$ expresando estos resultados una diferencia muy significativa. Esto nos indica que la parte superior de las extremidades (brazo, muslo) son parámetros más confiables para la medición directa antropométrica fetal.

De los parámetros estudiados, aquellos que demostraron una diferencia menos significativa (pero siempre siendo $\mathrm{P}<0,005)$ fueron:

- Vertex-coxis (semana 15) $\mathrm{P}=0,01$ (tabla 3)

- Circunferencia cefálica (semana 15) $\mathrm{P}=$ 0,03 (tabla 4)

- Occipito-frontal (semana 20) $P=0,048$ (tabla 5)

- Biparietal (semana 20) $P=0,005$ (tabla 6)

- Toraxico transverso (semana 21) $\mathrm{P}=$ 0,048 (tabla 7)

- Circunferencia abdominal (semana 20) P $=0,003$ (tabla 8)

- Antebrazo (semana 20) P = 0,002 (tabla 9)

- $\quad$ Mano (semana 21) $P=0,001$ (tabla 10)
- $\quad$ Pierna (semana 21) $\mathrm{P}=0,039$ (tabla 11)

- $\quad$ Pie (semana 21) $\mathrm{P}=0,032$ (tabla 12)

- Mentón-vertex (semana 20) $P=0,004$ (tabla 13)

Los parámetros sobre los cuales se aplicó (a través del programa estadístico) el test de hipótesis para la igualdad de varianza (test de Levene) fueron:

- Vertex-coxis: semana 15, 16, 17, 20

- Circunferencia cefálica: semana 15, 16, 17

- Occipito-frontal: semana 14, 15

- Biparietal: semana 14,15

- Toraxico transverso: semana 14, 16, 17, 18,19

- Circunferencia abdominal: semana 14 , $15,17,18,20$

- Brazo: semana 14, 18, 19

- Antebrazo: semana 14, 15, 18

- Mano: semana 14, 15, 16, 17

- Muslo: semana 14

- Pierna: semana 14, 15, 19, 20

- Pie: semana 14

- Mentón-vertex: semana 14

\begin{tabular}{|c|c|c|c|c|c|c|c|c|}
\hline \multirow{3}{*}{$\begin{array}{l}\text { Edad gestacional } \\
\quad \text { (semanas) }\end{array}$} & \multicolumn{8}{|c|}{ Circunferencia cefalica } \\
\hline & \multicolumn{3}{|c|}{ USG } & \multicolumn{3}{|c|}{ Hansmann } & \multirow{2}{*}{$\mathrm{T}$} & \multirow{2}{*}{$P$} \\
\hline & $\mathrm{n}$ & $\mathrm{M}$ & $\mathrm{S}$ & $\mathrm{n}$ & $\mathrm{M}$ & $\mathrm{S}$ & & \\
\hline 14 & 10 & 10,629 & ,305 & 13 & 9,490 & ,240 & 10,019 & ,000 \\
\hline 15 & 6 & 11,083 & , 129 & 8 & 10,498 & ,381 & 4,036 & ,003 \\
\hline 16 & 9 & 11,725 & ,286 & 5 & 11,190 & 045 & 5,488 & ,000 \\
\hline 17 & 9 & 13,458 & ,645 & 8 & 11,501 & ,270 & 8,311 & ,000 \\
\hline 18 & 9 & 15,706 & ,347 & 9 & 11,942 & ,265 & 25,824 & ,000 \\
\hline 19 & 6 & 16,048 & ,191 & 7 & 12,774 & ,261 & 25,332 & ,000 \\
\hline 20 & 4 & 16,350 & ,474 & 5 & 13,096 & ,188 & 14,198 & ,000 \\
\hline 21 & 7 & 16,828 & ,526 & 5 & 13,528 & ,243 & 12,935 & ,000 \\
\hline
\end{tabular}

Tabla 4: USG: ultrasonografía, $\mathbf{n}$ : $\mathrm{N}^{\circ}$ de individuos, M: Media, S: Desvío Standard. T: Prueba $t$ de Student, P: Probabilidad. Numeración en rojo: valores a los que se les aplicó el test de Levene 


\begin{tabular}{|c|c|c|c|c|c|c|c|c|}
\hline \multirow{2}{*}{$\begin{array}{c}\text { Edad gestacional } \\
(\text { semanas })\end{array}$} & \multicolumn{9}{|c|}{ Occipito - frontal } \\
\cline { 2 - 8 } & \multicolumn{3}{|c|}{ USG } & \multicolumn{3}{c|}{ Hansmann } & T \\
\cline { 2 - 8 } & $\mathrm{n}$ & $\mathrm{M}$ & $\mathrm{S}$ & $\mathrm{n}$ & $\mathrm{M}$ & $\mathrm{S}$ & & \\
\hline 14 & 10 & 3,576 &, 250 & 13 & 2,324 &, 125 & 14,441 &, 000 \\
\hline 15 & 6 & 3,956 &, 346 & 8 & 2,505 &, 144 & 9,650 &, 000 \\
\hline 16 & 9 & 4,290 &, 113 & 5 & 2,962 &, 088 & 22,541 &, 000 \\
\hline 17 & 9 & 4,791 &, 359 & 8 & 3,548 &, 386 & 6,875 &, 000 \\
\hline 18 & 9 & 5,172 &, 199 & 9 & 4,198 &, 209 & 10,108 &, 000 \\
\hline 19 & 6 & 5,571 &, 177 & 7 & 4,544 &, 187 & 10,095 &, 000 \\
\hline 20 & 4 & 5,8500 &, 163 & 5 & 5,262 &, 138 & 2,386 &, 048 \\
\hline 21 & 7 & 6,024 &, 346 & 5 & 5,324 &, 279 & 6,577 &, 000 \\
\hline
\end{tabular}

Tabla 5: USG: ultrasonografía, $\mathbf{n}: \mathrm{N}^{\circ}$ de individuos, $\mathbf{M}$ : Media, S: Desvío Standard. T: Prueba $\mathrm{t}$ de Student, P: Probabilidad. Numeración en rojo: valores a los que se les aplicó el test de Levene

\begin{tabular}{|c|c|c|c|c|c|c|c|c|}
\hline \multirow{3}{*}{$\begin{array}{l}\text { Edad gestacional } \\
\text { (semanas) }\end{array}$} & \multicolumn{8}{|c|}{ Bi parietal } \\
\hline & \multicolumn{3}{|c|}{ USG } & \multicolumn{3}{|c|}{ Hansmann } & \multirow{2}{*}{$T$} & \multirow[t]{2}{*}{$P$} \\
\hline & $\mathrm{n}$ & $M$ & $\mathrm{~S}$ & $\mathrm{n}$ & $M$ & $S$ & & \\
\hline 14 & 10 & 3,021 & ,331 & 13 & 1,150 & ,087 & 17,382 & ,000 \\
\hline 15 & 6 & 3,113 & ,038 & 8 & 1,468 & 162 & 27,564 & ,000 \\
\hline 16 & 9 & 3,292 & ,085 & 5 & 1,728 & ,054 & 36,501 & ,000 \\
\hline 17 & 9 & 4,154 & ,584 & 8 & 2,218 & ,316 & 8,332 & ,000 \\
\hline 18 & 9 & 4,166 & ,265 & 9 & 2,837 & , 194 & 12,134 & ,000 \\
\hline 19 & 6 & 4,245 & ,063 & 7 & 3,120 & 193 & 13,534 & ,000 \\
\hline 20 & 4 & 4,427 & ,343 & 5 & 3,700 & 183 & 4,100 & ,005 \\
\hline 21 & 7 & 5,032 &, 550 & 5 & 3,392 & ,255 & 6,143 & ,000 \\
\hline
\end{tabular}

Tabla 6: USG: ultrasonografía, $\mathbf{n}$ : $\mathrm{N}^{\circ}$ de individuos, $\mathbf{M}$ : Media, S: Desvío Standard. T: Prueba t de Student, P: Probabilidad. Numeración en rojo: valores a los que se les aplicó el test de Levene 


\begin{tabular}{|c|c|c|c|c|c|c|c|c|}
\hline \multirow{2}{*}{$\begin{array}{c}\text { Edad gestacional } \\
\text { (semanas) }\end{array}$} & \multicolumn{7}{|c|}{ Torácico transverso } \\
\cline { 2 - 8 } & $\mathrm{n}$ & $\mathrm{M} G$ & $\mathrm{~S}$ & $\mathrm{n}$ & $\mathrm{M}$ & $\mathrm{S}$ & $\mathrm{T}$ & $\mathrm{P}$ \\
\cline { 2 - 9 } & 10 & 2,845 &, 360 & 13 & 2,070 &, 060 & 6,730 &, 000 \\
\hline 14 & 6 & 3,166 &, 044 & 8 & 2,362 &, 126 & 14,787 &, 000 \\
\hline 15 & 9 & 3,538 &, 140 & 5 & 2,666 &, 037 & 17,542 &, 000 \\
\hline 16 & 9 & 3,977 &, 751 & 8 & 3,102 &, 319 & 3,185 &, 009 \\
\hline 17 & 9 & 4,151 &, 243 & 9 & 3,214 &, 080 & 10,976 &, 000 \\
\hline 19 & 6 & 4,385 &, 169 & 7 & 3,328 &, 448 & 5,775 &, 000 \\
\hline 20 & 4 & 4,122 &, 201 & 5 & 3,400 &, 332 & 4,025 &, 006 \\
\hline 21 & 7 & 5,232 &, 315 & 5 & 4,844 &, 260 & 2,255 &, 048 \\
\hline
\end{tabular}

Tabla 7: USG: ultrasonografía, $\mathbf{n}$ : $\mathrm{N}^{\circ}$ de individuos, M: Media, S: Desvío Standard.T: Prueba t de Student, P: Probabilidad. Numeración en rojo: valores a los que se les aplicó el test de Levene

\begin{tabular}{|c|c|c|c|c|c|c|c|c|}
\hline \multirow{3}{*}{$\begin{array}{l}\text { Edad gestacional } \\
\text { (semanas) }\end{array}$} & \multicolumn{8}{|c|}{ Circunferencia abdominal } \\
\hline & \multicolumn{3}{|c|}{ USG } & \multicolumn{3}{|c|}{ Hansmann } & \multirow{2}{*}{$\mathrm{T}$} & \multirow{2}{*}{$P$} \\
\hline & $\mathrm{n}$ & $\mathrm{M}$ & $S$ & $\mathrm{n}$ & $\mathrm{M}$ & $S$ & & \\
\hline 14 & 10 & 9,032 & 659 & 13 & 7,257 & ,250 & 8,066 & , 000 \\
\hline 15 & 6 & 10,298 & 255 & 8 & 7,806 & 105 & 22,487 & , 000 \\
\hline 16 & 9 & 10,558 & 146 & 5 & 8,230 & 183 & 26,101 & ,000 \\
\hline 17 & 9 & 11,177 & 207 & 8 & 9,596 & ,562 & 7,514 & , 000 \\
\hline 18 & 9 & 11,570 & 156 & 9 & 10,574 & 491 & 5,796 & , 000 \\
\hline 19 & 6 & 11,893 & 246 & 7 & 11,217 & 108 & 6,219 & 001 \\
\hline 20 & 4 & 13,302 & 326 & 5 & 12,150 & ,132 & 6,641 & ,003 \\
\hline 21 & 7 & 13,650 & ,260 & 5 & 12,440 & , 336 & 7,043 & , 000 \\
\hline
\end{tabular}

Tabla 8: USG: ultrasonografía, $\mathbf{n}$ : $\mathrm{N}^{\circ}$ de individuos, M: Media, S: Desvío Standard. T: Prueba t de Student, P: Probabilidad. Numeración en rojo: valores a los que se les aplicó el test de Levene. 


\begin{tabular}{|c|c|c|c|c|c|c|c|c|}
\hline \multirow{2}{*}{$\begin{array}{c}\text { Edad gestacional } \\
\text { (semanas) }\end{array}$} & \multicolumn{7}{|c|}{ Antebrazo } \\
\cline { 2 - 9 } & $\mathrm{n}$ & $\mathrm{M}$ & $\mathrm{S}$ & $\mathrm{n}$ & $\mathrm{M}$ & $\mathrm{S}$ & $\mathrm{T}$ & $\mathrm{P}$ \\
\cline { 2 - 9 } & 10 & 2,298 &, 170 & 13 & 1,387 &, 056 & 16,225 &, 000 \\
\hline 14 & 6 & 2,743 &, 093 & 8 & 1,726 &, 227 & 11,427 &, 000 \\
\hline 15 & 9 & 3,427 &, 094 & 5 & 2,148 &, 040 & 28,563 &, 000 \\
\hline 16 & 9 & 3,906 &, 444 & 8 & 2,197 &, 279 & 9,343 &, 000 \\
\hline 18 & 9 & 3,962 &, 243 & 9 & 2,648 &, 130 & 14,280 &, 000 \\
\hline 19 & 6 & 4,030 &, 062 & 7 & 2,875 &, 156 & 16,870 &, 000 \\
\hline 20 & 4 & 4,217 &, 063 & 5 & 3,472 &, 252 & 6,342 &, 002 \\
\hline 21 & 7 & 4,485 &, 249 & 5 & 3,932 &, 147 & 4,398 &, 001 \\
\hline
\end{tabular}

Tabla 9: USG: ultrasonografía, $\mathbf{n}$ : $\mathrm{N}^{\circ}$ de individuos, M: Media, S: Desvío Standard. T: Prueba $t$ de Student, P: Probabilidad. Numeración en rojo: valores a los que se les aplicó el test de Levene.

\begin{tabular}{|c|c|c|c|c|c|c|c|c|}
\hline \multirow{3}{*}{$\begin{array}{l}\text { Edad gestacional } \\
\text { (semanas) }\end{array}$} & \multicolumn{8}{|c|}{ Mano } \\
\hline & \multicolumn{3}{|c|}{ USG } & \multicolumn{3}{|c|}{ Hansmann } & \multirow{2}{*}{$\mathrm{T}$} & \multirow{2}{*}{$P$} \\
\hline & $\mathrm{n}$ & $M$ & $S$ & $n$ & $M$ & $S$ & & \\
\hline 14 & 10 & 1,213 & ,086 & 13 & ,953 & ,019 & 9,353 & , 000 \\
\hline 15 & 6 & 1,510 & ,071 & 8 & 9875, & ,012 & 17,743 & ,000 \\
\hline 16 & 9 & 1,863 & 139 & 5 & 1,078 & ,019 & 16,611 & , 000 \\
\hline 17 & 9 & 1,993 & 238 & 8 & 1,156 & 048 & 10,307 & ,000 \\
\hline 18 & 9 & 2,368 & 136 & 9 & 1,238 & 159 & 16,153 & ,000 \\
\hline 19 & 6 & 2,678 & 290 & 7 & 1,720 & 191 & 7,127 & ,000 \\
\hline 20 & 4 & 3,185 & 203 & 5 & 2,250 & ,122 & 8,599 & ,000 \\
\hline 21 & 7 & 3,476 & ,317 & 5 & 2,720 & , 182 & 4,755 & ,001 \\
\hline
\end{tabular}

Tabla 10: USG: ultrasonografía, $\mathbf{n}$ : $\mathrm{N}^{\circ}$ de individuos, M: Media, S: Desvío Standard. T: Prueba $\mathrm{t}$ de Student, P: Probabilidad. Numeración en rojo: valores a los que se les aplicó el test de Levene 


\begin{tabular}{|c|c|c|c|c|c|c|c|c|}
\hline \multirow{2}{*}{$\begin{array}{c}\text { Edad gestacional } \\
\text { (semanas) }\end{array}$} & \multicolumn{7}{|c|}{ USG } & \multicolumn{7}{c|}{ Hansmann } \\
\cline { 2 - 8 } & $\mathrm{n}$ & $\mathrm{M}$ & $\mathrm{S}$ & $\mathrm{n}$ & $\mathrm{M}$ & $\mathrm{S}$ & $\mathrm{T}$ & $\mathrm{P}$ \\
\hline 14 & 10 & 2,107 &, 373 & 13 & 1,286 &, 037 & 6,911 &, 000 \\
\hline 15 & 6 & 2,573 &, 401 & 8 & 1,468 &, 104 & 6,582 &, 001 \\
\hline 16 & 9 & 3,302 &, 135 & 5 & 1,838 &, 042 & 23,125 &, 000 \\
\hline 17 & 9 & 4,038 &, 278 & 8 & 2,327 &, 239 & 13,470 &, 000 \\
\hline 18 & 9 & 4,615 &, 246 & 9 & 2,895 &, 139 & 18,234 &, 000 \\
\hline 19 & 6 & 4,725 &, 094 & 7 & 3,668 &, 410 & 6,607 &, 000 \\
\hline 20 & 4 & 4,892 &, 025 & 5 & 4,266 &, 074 & 17,634 &, 000 \\
\hline 21 & 7 & 5,437 &, 339 & 5 & 5,018 &, 233 & 2,374 &, 039 \\
\hline
\end{tabular}

Tabla 11: USG: ultrasonografía, $\mathbf{n}$ : $\mathrm{N}^{\circ}$ de individuos, M: Media, S: Desvío Standard. T: Prueba $t$ de Student, P: Probabilidad. Numeración en rojo: valores a los que se les aplicó el test de Levene

\begin{tabular}{|c|c|c|c|c|c|c|c|c|}
\hline \multirow{3}{*}{$\begin{array}{l}\text { Edad gestacional } \\
\text { (semanas) }\end{array}$} & \multicolumn{8}{|c|}{ Pie } \\
\hline & \multicolumn{3}{|c|}{ USG } & \multicolumn{3}{|c|}{ Hansmann } & \multirow{2}{*}{$\mathrm{T}$} & \multirow{2}{*}{$P$} \\
\hline & $\mathrm{n}$ & $M$ & $S$ & $\mathrm{n}$ & $\mathrm{M}$ & $S$ & & \\
\hline 14 & 10 & 1,230 & 16310, & 13 & 9515 & 03508, & 5,306 & ,000 \\
\hline 15 & 6 & 1,598 & ,07360 & 8 & 1,0238 & 03852, & 19,039 & ,000 \\
\hline 16 & 9 & 1,950 & 04416, & 5 & 1,1360 & 03050, & 36,371 & ,000 \\
\hline 17 & 9 & 1,985 & 22716 & 8 & 1,2700 & 10085, & 8,198 & ,000 \\
\hline 18 & 9 & 2,817 & 32139, & 9 & 1,5911 & 14075, & 10,488 & ,000 \\
\hline 19 & 6 & 2,868 & ,03656 & 7 & 1,9171 & 26139, & 9,520 & ,000 \\
\hline 20 & 4 & 2,997 & 13961, & 5 & 2,6800 & 09695, & 4,040 & ,005 \\
\hline 21 & 7 & 3,370 & ,357 & 5 & 2,928 & 194 & 2,489 & ,032 \\
\hline
\end{tabular}

Tabla 12: USG: ultrasonografía, $\mathbf{n}: \mathrm{N}^{\circ}$ de individuos, $\mathbf{M}$ : Media, S: Desvío Standard. T: Prueba $\mathrm{t}$ de Student, P: Probabilidad. Numeración en rojo: valores a los que se les aplicó el test de Levene. 


\begin{tabular}{|c|c|c|c|c|c|c|c|c|}
\hline \multirow{2}{*}{$\begin{array}{c}\text { Edad gestacional } \\
\text { (semanas) }\end{array}$} & \multicolumn{7}{|c|}{ Mentón-vertex } \\
\cline { 2 - 8 } & $\mathrm{n}$ & $\mathrm{M}$ & $\mathrm{S}$ & $\mathrm{n}$ & $\mathrm{M}$ & $\mathrm{S}$ & $\mathrm{T}$ & $\mathrm{P}$ \\
\hline 14 & 10 & 3,091 &, 369 & 13 & 2,286 &, 070 & 6,796 &, 000 \\
\hline 15 & 6 & 3,770 &, 075 & 8 & 2,578 &, 125 & 20,518 &, 000 \\
\hline 16 & 9 & 4,421 &, 094 & 5 & 2,782 &, 047 & 35,953 &, 000 \\
\hline 17 & 9 & 4,770 &, 290 & 8 & 3,200 &, 348 & 10,125 &, 000 \\
\hline 18 & 9 & 5,421 &, 095 & 9 & 3,776 &, 129 & 30,707 &, 000 \\
\hline 19 & 6 & 5,850 &, 056 & 7 & 4,245 &, 173 & 21,549 &, 000 \\
\hline 21 & 4 & 6,027 &, 532 & 5 & 4,958 &, 206 & 4,175 &, 004 \\
\hline & 7 & 6,475 &, 372 & 5 & 5,028 &, 104 & 9,772 &, 000 \\
\hline
\end{tabular}

Tabla 13: USG: ultrasonografía, $\mathbf{n}$ : $\mathrm{N}^{\circ}$ de individuos, $\mathbf{M}$ : Media, S: Desvío Standard. T: Prueba $t$ de Student, P: Probabilidad. Numeración en rojo: valores a los que se les aplicó el test de Levene

\section{DISCUSIÓN}

El crecimiento humano es un proceso que se extiende desde la vida intrauterina hasta la edad adulta. La multiplicidad de factores implicados en este proceso hace que su valoración constituya un indicador sensible, aunque no específico, del estado de salud y bienestar de un sujeto o de una comunidad (Eveleth, 1990).

Existe un incremento progresivo de los parámetros antropométricos evaluados a medida que avanza la duración de la gestación (Carrascosa, 2004).

Mucho se ha estudiado sobre la manera de determinar la edad gestacional. La edad gestacional de un feto puede ser un problema forense importante, por ello varios investigadores han realizado fórmulas basadas en la longitud vertex-talón, longitud vertex-coxis o los diámetros del cuerpo (Warren, 1999).

El diagnóstico de la edad del feto humano es generalmente estimado a partir de la medición de la longitud vertex-coxis o la longitud vertex-talón y el peso del feto. Sin embargo, estas mediciones no son totalmente exactas y a veces es necesario combinarlas con otros datos para determinar la edad del feto (Lizardo-Daudt, 2002).
El diámetro biparietal es sólo una medida que puede tomarse del feto a fin de estimar la edad gestacional. Según Deter et al (1982), no ser capaz de obtener una medición de este parámetro en el momento de un examen de ultrasonido indicaría no haber realizado un trabajo adecuado. Sin embargo, reconocer la variabilidad en la forma de la cabeza fetal, cambió la concepción sobre el valor de esta medida en la estimación de la edad gestacional, especialmente después de las 20 semanas de gestación. Por ello se impulsa la medición de varios segmentos anatómicos, lo que se ha denominado biometría fetal. Se ha encontrado que la medición de más de un parámetro fetal disminuye el margen de error propio de cada uno de ellos. (Hohler, 1984)

Diversas mediciones sobre la superficie externa de la estructura corporal son útiles para estimar la edad fetal. La longitud vertex-coxis es la medida más utilizada, aunque la talla de los fetos varía considerablemente para la misma edad. La longitud del pie se correlaciona bien con la longitud vertex-coxis y es particularmente útil para estimar la edad de fetos incompletos 0 macerados. (Moore, 1996). 
La edad gestacional puede ser determinada a partir de la medición ecográfica de varias estructuras, pero la más utilizada es la longitud del fémur (Maroun, 2005), por ser el parámetro considerado más fidedigno. Por esta razón utilizamos este criterio en el presente trabajo.

El diámetro biparietal mantiene la correlación más estrecha con la edad gestacional en el segundo trimestre (Degani, 2001), pero las variaciones en la forma del cráneo del feto pueden afectar negativamente su exactitud. En estos casos, la circunferencia cefálica tiene la ventaja de ser independiente de la forma del cráneo y puede ser utilizada como un medio alternativo para establecer la edad gestacional (Hadlock, 1981). Debido a estas discrepancias entre distintos autores con respecto a los parámetros más fidedignos es que no nos limitamos a utilizar un solo criterio de medición, sino que utilizamos varios para disminuir el margen de error.

Las dimensiones fetales obtenidas ecográficamente se aproximan mucho a las medidas de longitud vertex-coxis. (Moore, 1996)

La amplia variabilidad que muestran los parámetros antropométricos en relación a factores raciales, genéticos, sociales, ambientales y estilos de vida maternos, hacen aconsejable que cada comunidad disponga de sus propias tablas de crecimiento intrauterino, así como la necesidad de revisarlas periódicamente (Carrascosa, 2004; Fok, 2003).

Los patrones de crecimiento intrauterino deben estar sujetos a actualización constante debido a que los cambios sociales, económicos, de los estilos de vida y hábitos nutricionales maternos parecen ejercer importantes influencias en las características antropométricas de los recién nacidos (Kramer, 2001)

Existen diferencias antropométricas étnicas. Estas diferencias entre razas puede aumentar el error en la estimación del estadio fetal si las fórmulas se han obtenido a partir de muestras de razas diferentes a la de la población que se utilizó como objeto del estudio. Así, en un estudio realizado en distintas poblaciones, no hubo diferencia significativa en la relación entre la longitud vertex-talón y diáfisis del fémur para las poblaciones malayas y chinas, pero la relación fue significativamente diferente en la población indígena. Para una longitud dada de la diáfisis del fémur, la longitud vertex-talón de la población indígena fue, en promedio, $1,1 \mathrm{~cm}$ más corta que la longitud vertex-talón de la población malaya y china. (Lim, 2000).

La variación en la biometría fetal entre los diferentes grupos étnicos se ha reportado mayormente durante el segundo trimestre
(Jacquemyn, 2000; Thame, 2003, Mastrobattista, 2004; Paladini, 2005; Salomon 2006).

Varios autores recomiendan establecer rangos de referencia a partir de poblaciones locales para garantizar así que la muestra sea realmente representativa (Bhat, 1989; Larroche, 1997).

En relación a estas variaciones, se ha demostrado que la genética del feto incide con mayor importancia sobre la longitud del mismo y la circunferencia cefálica. (Lunde, 2007). Además, los estándares de crecimiento fetal pueden utilizarse para la identificación del recién nacido cuyo desarrollo se aleja de los patrones normales de crecimiento y que, por ende, está expuesto a una mayor morbilidad y mortalidad durante el periodo neonatal y la edad adulta (Carrascosa, 2004).

Es importante trabajar mejorando los patrones de crecimiento, para que así puedan ser utilizados en una amplia variedad de situaciones (Cole, 1996), una de ellas la aplicación científica.

Nuestro estudio no pretendió desarrollar una metodología nueva, ni un criterio que salvara las diferencias mencionadas, sino sólo comparar las dos mediciones utilizadas con mayor frecuencia. Como resultado de ello concluímos que ambas modalidades de medición son diferentes para una misma edad gestacional $y$, por ende, resultaría más apropiado referirse a fetos con ciertas dimensiones según alguno de estos parámetros que a "edad gestacional", a menos que se cuente con criterios locales de valoración.

\section{BIBLIOGRAFÍA}

Bath GJ, Mukelabai K, Shastri GN. 1989. Anthropometric parameters of Zambian infants at birth. J Trop Pediat 35: 100-4.

Carlson B. 2005. Embriología humana y biología del desarrollo. $3^{a}$ Edición, España: Editorial Elseiver, pag. 477-8.

Carrascosa A, Yeste D, Copil A, Almar J, Salcedo S, Gussinyé M. 2004. Patrones antropométricos de los recién nacidos pretermino y a término en el Hospital Maternoinfantil Vall D'Hebron. An Pediatr 60: 406-16.

Cole TJ. 1996. Some questions about how growth standards are used. Horm Res 45: 1823.

Degani S. 2001. Fetal biometry: clinical, pathological and technical considerations. Obstetrical and gynecological survey 56: 159 67.

Deter RL, Harrist RB, Hadlock FP, Poindexter $A N$. 1982. Longitudinal studies of fetal growth with the use of dynamic image 
ultrasonography. Am J Obstet Gynecol 143: 545-54

Eveleth PB, Tanner JM. 1990. Worldwide variation in human growth. $2^{\mathrm{a}}$ Edición, Cambridge: Cambridge University Press, pag 15.

Fok TF, So HK, Wong E, Ng PC, Chang A, Lau J, Chow CB, Lee WH. 2003. Update gestational age specific birth weight, crown-heel length, and head circumference of Chinese newborns. Arch Dis Child Fetal Neonatal 88: 229-36.

Hadlock FP, Deter RL, Carpenter RL. 1981. Estimating fetal age: effects of head shape on BPD. Am J Roentgenol 137: 83-85.

Hansmann M, Schuhmacher H, Foebus J, Voigt $U$. 1979. Ultrasonic biometry of the fetal crown-rump length between 7 and 20 weeks gestation. Geburtshilfe Frauenheilkd 39: 65666.

Hobler CW. 1984. Ultrasound estimation of gestational age. Clin Obstet Gynecol 27: 31426.

Jacquemin Y, Sys SU, Verdonk P. 2000. Fetal transverse cerebellar diameter in different ethnic groups. J Perinat Med 28: 14-19.

Kramer MS, Morin I, Yang H, Platt RW, Usher R, McNamara H. 2002. Why are babies getting bigger? Temporal trends in fetal growth and its determinants. J Pediatr 141: 538-42.

Lapunzina $P$, Aiello $H$. 2002. Manual de antropometría normal y patológica: fetal, neonatal, niños y adultos. $1^{\mathrm{a}}$ Edición, Barcelona: Editorial Masson, pag 318-9.

Larroche JC. 1997. Post-mortem examination. Developmental pathology of the neonate. $1^{\mathrm{a}}$ Edición, Amsterdam: Excepta Medica, pag 1521.

Leung TN, Pang MW, Daljit SS, Leung TY, Poon CF, Wong SM, Lau TK. 2008. Fetal biometry in ethnic Chinese: biparietal diameter, head circumference, abdominal circumference and femur length. Ultrasound Obstet Gynecol 31: 321-7.

Lim JM, Hong AG, Raman S, Shymala N. 2000. Relationship between fetal femur diaphysis length and neonatal crown-heel length: the effect of race. Ultrasound Obstet Gynecol 15: 131-7.

Lizardo-Daudt HM, Albano Edelweiss MI, Teixeira dos Santos F, Alves Schumacher $R$. 2002. Diagnosis of the human fetal age based on the development of the normal kidney. Jornal Brasileiro de Patologia e Medicina Laboratorial 38: 135-9.

Lunde A, Melve KK, Gjessing HK, Skjaerven R, Irgens LM. 2007. Genetic and environmental influences on birth weight, birth length, head circumference, and gestational age by use of population-based parent-offspring data. Am J Epidemiol 165: 134-41.

Maroun LL, Graem N. 2005. Autopsy standards of body parameters and fresh organ weigths in nonmacerated and macerated human fetuses. Pediatr Dev Pathol 8: 204-17.

Mastrobattista JM, Pschirrer FR, Hamrick MA. 2004. Humerus length evaluation in different ethnic groups. J Ultrasound Med 23: 227-31.

Moore K, Persaud TVN, Shiota K. 1996. Atlas de Embriología Clínica. $1^{\text {a }}$ Edición, Madrid: Editorial Médica Panamericana, pag 63-65.

Paladini D, Rustico M, Viora E. 2005. Fetal size charts for the Italian population. Normative curves of head, abdomen and long bones. Prenat Diagn 25: 456-64.

Sadler TW. 2003. Langman's Medical Embriology. 9a Edition, Montana: Editorial Lippincott Williams \& Wilkins, pag 121.

Salomon LI, Duime M, Crequat J, Brodaty G, Talmant C, Fries N. 2006. French fetal biometry: reference equations and comparison with other charts. Ultrasound Obstet Gynecol 28: 193-98.

Thame M, Osmond C, Fletcher H, Forrester TE. 2003. Ultrasound derived fetal growth curves for a Jamaican population. West Indian Med 52: 99-110.

Warren MW. 1999. Radiographic determination of developmental age in fetuses and stillborns. J Forensic Sci 44: 708-12. 